\title{
A Retrospective Assessment of 741 Patients with Sudden Hearing Loss
}

\author{
M. Canis ${ }^{1}$, D. Osterkorn ${ }^{2}$, K. Osterkorn ${ }^{2}$ and M. Suckfuell ${ }^{*}, 1$ \\ ${ }^{I}$ Department of Otorhinolaryngology, Head and Neck Surgery, University of Munich, Germany \\ ${ }^{2}$ Medizinisches Wirtschaftsinstitut GmbH, Munich, Germany
}

\begin{abstract}
Background: Sudden hearing loss is a result of various factors. Most therapies aim to improve the cochlear microcirculation but effectiveness is not sufficiently documented.

Methods: 741 patients were included into the non-interventional observation thus far and analyzed with regard to various therapies and time progressions. The main outcome measure consisted of hearing threshold difference before, immediately after and 90 days after therapy.

Results: In a summary of all evaluable patients $(\mathrm{n}=630)$, a hearing increase of $14 \mathrm{~dB}$ was shown after therapy. Evaluation of remission rates regarding time between occurrence and therapy $(n=547)$ showed hearing increase of $14 d B(n=444)$ if therapy was started within 2 days, $12 \mathrm{~dB}(\mathrm{n}=56)$ between day 2 and day $7,13 \mathrm{~dB}(\mathrm{n}=18)$ between 1 and 2 weeks, $6 \mathrm{~dB}$ $(n=23)$ between 2 and 6 weeks, and $13 \mathrm{~dB}(n=6)$ after more than 6 weeks. In consideration of the total hearing loss, an improvement of hearing loss from $29 \mathrm{~dB}$ to $16 \mathrm{~dB}$ was obtained after therapy with HES $(\mathrm{n}=115)$ immediately after therapy, remaining constant up to day 90 . After sole therapy with cortisone $(n=33)$, hearing loss improved from $31 \mathrm{~dB}$ to $17 \mathrm{~dB}$ with constancy on day 90 . If patients were treated with combination therapy consisting of HES and cortisone $(n=206)$, an improvement from $31 \mathrm{~dB}$ to $18 \mathrm{~dB}$ after termination of therapy and of $17 \mathrm{~dB}$ after day 90 was shown in the consideration of total hearing loss.

Discussion: Idiopathic hearing loss is a heterogenic illness; evaluation of different therapies in different forms of hearing loss will have importance in the future. The retrospective assessment is not limited by time and will use its large case numbers to identify subgroups of hearing loss which can be used to define criteria for a more specific and differential therapy.
\end{abstract}

\section{INTRODUCTION}

With an estimated incidence of approximately 290 new cases per 100000 residents per year, hearing loss is one of the most frequent ear disorders in western countries [1]. Even though there is often at least partial remission, lasting hearing loss and tinnitus are problematic consequences in many cases. The pathogenesis of this illness is still largely unknown even today, and is subject to controversial discussion. The most frequently discussed causes of hearing loss, also due to the clinical signs with sudden, one-sided commencement and possible spontaneous remission within hours or days, include disturbances in the cochlear microcirculation. Animal studies revealed that cochlear microcirculation is highly subject to disturbances and even slight changes in the regional blood flow may cause functional changes of the organ of Corti [2]. However, a monocausal occurrence of the illness is rather unlikely, so that numerous other causalities such as viral infections, immune and autoimmune pathological processes, endolymphatic hydrops or a sum of these mechanisms are under discussion [3]. Despite various experimental investigations, there are nonetheless few clinical data about hearing loss which were collected on large case numbers and methodologically satisfy the requirements of

\footnotetext{
*Address correspondence to this author at the Department of Otorhinolaryngology, Head and Neck Surgery, University of Munich, Marchioninistr. 15, D-81377 Munich, Germany; Tel: +49-89-7095-0; Fax: +49-89-
} 7095-6869; E-mail: markus.suckfuell@med.uni-muenchen.de evidence-based medicine. Among other things, this is due to the fact that too few patients come into clinics early enough, that they are either not treated at all or primarily by resident specialists on an outpatient basis and that clinical starting situation is very heterogenic. Numerous therapies of hearing loss are described in the hearing loss guidelines, however their efficacy was thus far demonstrated only for few procedures in clinical studies $[4,5]$. Plasma expanders such as hydroxyethyl starch (HES), dextrane and corticosteroids, individually or in combination, are one of the treatments most commonly used in Germany. Plasma expanders are intended to improve the rheological properties of the blood due to hemodilution. The effect of glucocorticosteroids is based on an anti-inflammatory and swelling-reducing effect with the assumption that an inflammation or immune system modulated reaction on the inner ear is causative for the development of the hearing loss. The effect which reduces swelling is also intended to increase cochlear blood flow. Aside from this, there are other more or less well examined procedures such as H.E.L.P. apheresis, hyperbaric oxygen therapy, ionotropic or anti-oxidative medications. H.E.L.P. apheresis is intended to bring about a considerable improvement in the flow characteristics of the blood by drastically lowering the serum cholesterol and plasma fibrinogen levels without hemodilution. It was shown in a clinical study that in sudden hearing loss, drastically lowering the LDL and fibrinogen levels through heparin induced, extracorporeal LDL precipitation (H.E.L.P. apheresis) produces a significantly better improvement in the hearing level as compared 
to the control group, which was treated with steroids [5]. Most therapeutic forms aim to improve the microcirculatory parameters in order to obtain a therapeutic benefit regarding hearing levels by improving the oxygen supply to the outer hair cells. However, the efficacy of nearly all therapies is currently not sufficiently proven and subject to controversial discussion. Due to the poor clinical data situation, it is therefore not possible to form sub-groups of hearing loss which could be used to define clearly delimitable criteria for differing therapeutic modalities. In order to answer these questions, 741 patients were included into a retrospective assessment thus far and analyzed with regard to various therapies and time progressions. Using the collected data, it is possible on the basis of a large number of patients to make more differentiated statements about the effectiveness of various therapeutic modalities in various types of hearing loss and differing time periods between the loss of hearing and the initial therapy.

\section{PATIENTS AND METHODS}

This retrospective assessment serves to document results and evaluate therapies in the treatment of hearing loss patients. All patients provided their consent prior to inclusion into the retrospective assessment. The monitoring and evaluation of collected data was performed by an independent statistics institute (Medizinisches Wirtschaftsinstitut $\mathrm{GmbH}$, Munich).

In the period from January $1^{\text {st }}, 2004$ to December $31^{\text {st }}$, 2006, a total of 741 patients with ages from 18 to 84 years were included at 40 therapy centers throughout Germany. The inclusion criterion consisted of one-sided, acute hearing loss with a duration of up to 2 months and without previous treatment.

Prior to treatment, medical history was collected from the patients regarding demographic data, general cardiovascular risk factors, the duration of the hearing loss, possible causal events (such as loud sound trauma or otitis media), accompanying tinnitus or rotatory vertigo. The audiometric examinations were performed before and immediately after therapy as well as at a follow-up examination 90 days after the end of the treatment. The examinations included pure tone audiometry at the frequencies of 125, 250,500,1000, 2000,3000,6000 and $8000 \mathrm{~Hz}$, implemented in accordance with ISO 7029, as well as tympanometry. Retrocochlear hearing disturbances were excluded by brainstem response audiometry or MRI. In patients with tinnitus, the subjective nuisance was determined using a visual analogue scale from 0 to 10 . Therapy-associated itching was documented in the case of occurrence at the follow-up examination. All data were documented by the treating physicians in a standardized form. The following sub-groups were formed with regard to the therapy: (1) HES total $(6 \%$ and $10 \%$ ); Cortisone IV ; (6) HES and cortisone; (7) other therapeutic forms. All therapies were conducted with respect to the sudden hearing loss guidelines. However, differences of therapy regarding doses and times between the centers have occurred.

\section{OUTCOME MEASURES, STATISTICAL EVALUA- TION}

This retrospective assessment is able - on the basis of a large patient collective - to make statements about the influ- ence which the type of hearing loss and the time period between hearing loss and start of therapy has on the remission rate in dependence on the therapeutic modality.

For this purpose, hearing levels were determined by means of pure tone audiometry, and determination of tinnitus nuisance before as well as immediately after and 90 days after termination of the therapy as described above. The main outcome measure consisted of the hearing level difference in pure tone audiometry $(0.25 ; 0.5 ; 1 ; 2 ; 4$ and $8 \mathrm{kHz})$ before as well as immediately after and 90 days after the therapy. In order to allow a recognition of any differences in the therapeutic response behavior and clinical progression, the pure sound audiograms were used to observe various sub-groups depending on the type of hearing loss: (1) low frequency [250 - $1000 \mathrm{~Hz}$; (2) middle frequency [1000 $4000 \mathrm{~Hz}$; (3) high frequency [2000 -8000] and (4) pantonal hearing loss $[250-8000 \mathrm{~Hz}]$.

Furthermore, time-related groups were formed in order to enable a statement about the remission rates in dependence of the time progression: $(1) \leq 2$ days; $(2)>2$ days and $\leq 1$ week; (3) $>1$ week and $\leq 2$ weeks; (4) $>2$ weeks and $\leq 6$ weeks; (5) $>6$ weeks. The following sub-groups were formed with regard to the therapy: (1) HES total $(6 \%$ and 10\%); (2) Cortisone IV ; (3) HES and cortisone; (3) other therapeutic forms.

The data were collected, checked for entry errors, validated and corrected. The statistical evaluation is descriptive and was performed with SAS (Version 9.1) for Windows and the software SPSS (Version 12.0). Due to the fact that no randomisation into the different groups occurred, confirming statistical tests were not used to show significance.

\section{RESULTS}

Table 1 shows the composition as well as the cardiocirculatory risk factors of the entire examined patient collective. After observation of the dataset of 741 patients, a total of 630 patients who were assignable to hearing loss in the low, middle and high frequency ranges as well as pantonal hearing loss remained.

The demographic data as well as the cardiocirculatory risk factors in dependence of the type and severity of hearing loss are shown in Table 2. Fig. (1) shows the evaluation of the main outcome measure prior to the start of therapy, immediately after therapy as well as 90 days after termination of the therapy. The evaluation initially took place depending on the type of hearing loss, without dependence of the time between the hearing loss and start of therapy, and without consideration of the therapeutic modalities. In consideration of the hearing loss in the low frequency range, the hearing loss prior to the start of therapy was $27 \mathrm{~dB}$. After completion of the therapy, the hearing loss improved to $14 \mathrm{~dB}$ and was approximately constant on day 90 with $12 \mathrm{~dB}$. For middle frequency hearing loss, a hearing loss of $23 \mathrm{~dB}$ before and 16 $\mathrm{dB}$ after therapy was found. This hearing improvement also remained constant at $16 \mathrm{~dB} 90$ days after the start of therapy. In hearing loss in the high frequency range, a hearing loss of $31 \mathrm{~dB}$ prior to therapy was found. In the post-therapeutic control, the improvement to a hearing loss of $21 \mathrm{~dB}$ was measured, this also remained constant at approximately 22 $\mathrm{dB}$ in the follow-up checks. In the consideration of pantonal hearing loss, a hearing loss of $37 \mathrm{~dB}$ was confirmed. Imme- 
diately after therapy and after 90 days, hearing loss improved to $19 \mathrm{~dB}$. In the summarization of all types of hearing loss, a pre-therapy hearing loss of $31 \mathrm{~dB}$ was found. After the end of therapy and in the 90 day follow-up examination, an improvement to $17 \mathrm{~dB}$ was obtained.

Table 1. The Composition as Well as the Cardiocirculatory Risk Factors of the Entire Examined Patient Collective

\begin{tabular}{|l|l|}
\hline \multicolumn{1}{|c|}{ Patients } & \\
\hline \hline $\begin{array}{l}\text { Gender } \\
\text { male } \\
\text { female } \\
\text { not reported }\end{array}$ & $305(48 \%)$ \\
\hline Hypertension & $303(48 \%)$ \\
\hline Nicotine consumption & $22(4 \%)$ \\
\hline Hyperlipedemia & $102(16 \%)$ \\
\hline Diabetes mellitus & $66(10 \%)$ \\
\hline Cardiovascular diseases (myocardial infarction, stroke) & $13(2 \%)$ \\
\hline Allergies & $25(4 \%)$ \\
\hline Skin diseases & $47(7 \%)$ \\
\hline Values as number of patients and percent. & $10(2 \%)$ \\
\hline
\end{tabular}

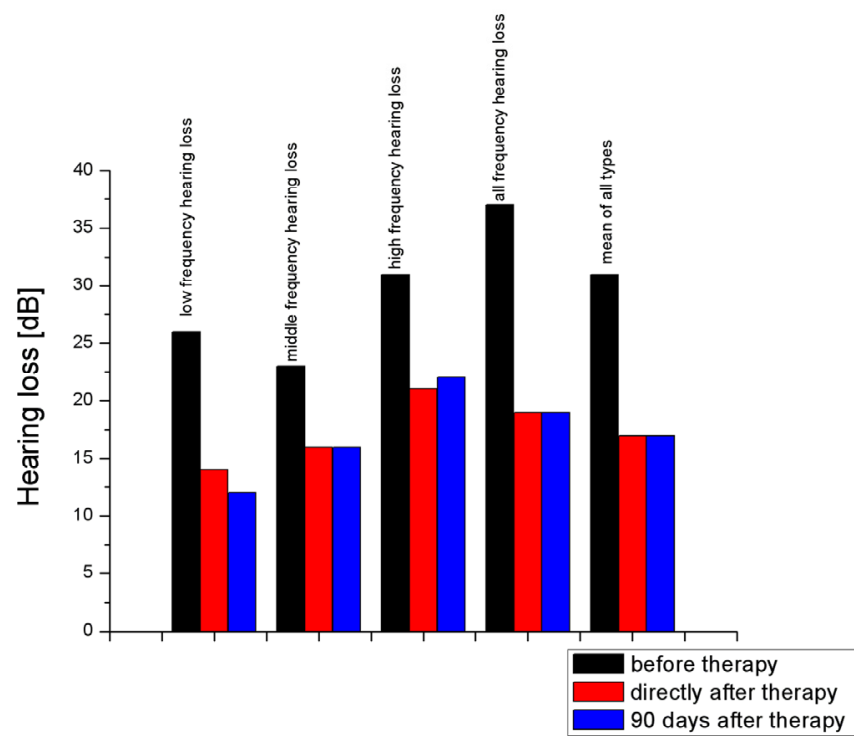

Fig. (1). The evaluation of the main outcome measure prior to the start of therapy, immediately after therapy as well as 90 days after termination of the therapy.

The evaluation of the remission rates with reference to the time interval between the occurred hearing loss and the respectively performed therapy is shown in Fig. (2). With therapy commencement within 2 days $(n=444)$ after the occurrence of the first symptoms of hearing loss, there was an improvement from $31 \mathrm{~dB}$ to $17 \mathrm{~dB}$ in the consideration of total hearing loss after the termination of therapy; this remained constant up to day 90 . In patients $(n=56)$ who were provided with therapy between day 2 and day 7 after the hearing loss event, the hearing loss in the pure sound audiogram improved from $30 \mathrm{~dB}$ to $18 \mathrm{~dB}$ after completion of the therapy. 90 days after the start of therapy, a slight improvement to $11 \mathrm{~dB}$ hearing loss was reached. If patients saw their doctor within 1 to 2 weeks after the hearing loss event $(n=18)$, an improvement in the hearing loss from 35 $\mathrm{dB}$ to $22 \mathrm{~dB}$ was obtained after therapy. In the follow-up check on day 90 after the start of therapy, a hearing loss of $19 \mathrm{~dB}$ was shown. Between 2 and 6 weeks after the start of symptoms $(n=23)$, a hearing gain of $6 \mathrm{~dB}$ after therapy end and $9 \mathrm{~dB}$ after 90 days was shown with a starting parameter of $24 \mathrm{~dB}$. In a time interval of more than 6 weeks between the hearing loss and commencement of therapy $(n=6)$, hearing loss improved from $23 \mathrm{~dB}$ to $10 \mathrm{~dB}$ with renewed worsening to $38 \mathrm{~dB}$ after 90 days.

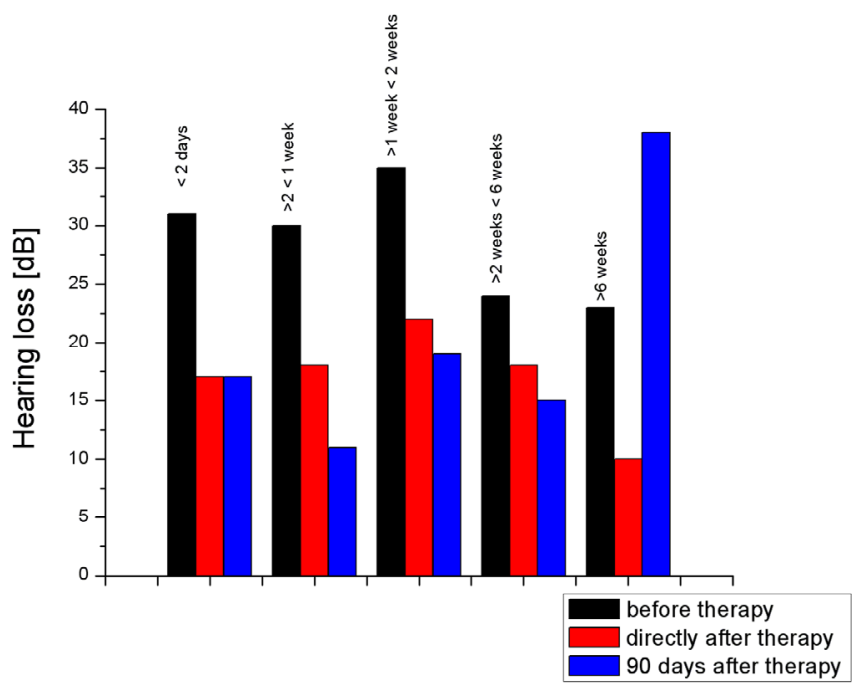

Fig. (2). The evaluation of the remission rates with reference to the time interval between the occurred hearing loss and the respectively performed therapy.

When describing the dependence of remission on therapeutic modalities, the groups HES total, Cortisone IV and HES in combination with cortisone will be compared. Fig. (3a-c) show the hearing loss of the various hearing loss types before and after therapy with the described therapeutic agents. In consideration of the total hearing loss, an improvement of hearing loss from $29 \mathrm{~dB}$ to $16 \mathrm{~dB}$ was obtained after therapy with HES ( $n=115)$ immediately after therapy, remaining constant up to day 90. After sole therapy with cortisone $(n=33)$, hearing loss improved from $31 \mathrm{~dB}$ to 17 $\mathrm{dB}$ with constancy on day 90 . If patients were treated with combination therapy consisting of HES and cortisone $(n=206)$, an improvement from $31 \mathrm{~dB}$ to $18 \mathrm{~dB}$ after termination of therapy and of $17 \mathrm{~dB}$ after day 90 was shown in the consideration of total hearing loss.

\section{DISCUSSION}

Little is known about the pathogenesis of idiopathic hearing loss; nonetheless, disturbances in the cochlear microcirculation are among the most commonly discussed causes. In a significant share of patients, the clinical signs of hearing loss are similar to those of other vascular disorders such as stroke or myocardial infarction. However, the association of hearing loss and vascular risk factors is still subject to controversial discussion. Nonetheless, numerous therapeutic 
Table 2. The Demographic Data as Well as the Cardiocirculatory Risk Factors in Dependence of the Type and Severity of Hearing Loss.

\begin{tabular}{|c|c|c|}
\hline $\begin{array}{l}\text { Type and Hearing } \\
\text { Loss }\end{array}$ & Patients & \\
\hline \multirow[t]{9}{*}{ Low Frequency } & $\begin{array}{l}\text { Gender } \\
\text { male } \\
\text { female } \\
\text { not reported }\end{array}$ & $\begin{array}{c}73(39 \%) \\
101(55 \%) \\
11(6 \%)\end{array}$ \\
\hline & hypertension & $30(16 \%)$ \\
\hline & nicotine consumption & $15(8 \%)$ \\
\hline & stress & $51(28 \%)$ \\
\hline & hyperlipedemia & $6(3 \%)$ \\
\hline & $\begin{array}{l}\text { Cardiovascular diseases } \\
\text { (myocardial infarction, stroke) }\end{array}$ & $2(1 \%)$ \\
\hline & diabetes mellitus & $7(4 \%)$ \\
\hline & allergies & $14(8 \%)$ \\
\hline & skin diseases & $1(1 \%)$ \\
\hline \multirow[t]{9}{*}{ Middle Frequency } & $\begin{array}{l}\text { Gender } \\
\text { male } \\
\text { female } \\
\text { not reported }\end{array}$ & $\begin{array}{c}43(42 \%) \\
56(55 \%) \\
3(3 \%) \\
\end{array}$ \\
\hline & hypertension & $15(15 \%)$ \\
\hline & nicotine consumption & $16(16 \%)$ \\
\hline & stress & $28(27 \%)$ \\
\hline & hyperlipedemia & $0(0 \%)$ \\
\hline & $\begin{array}{l}\text { Cardiovascular diseases } \\
\text { (myocardial infarction, stroke) }\end{array}$ & $2(2 \%)$ \\
\hline & diabetes mellitus & $2(2 \%)$ \\
\hline & allergies & $7(7 \%)$ \\
\hline & skin diseases & $1(1 \%)$ \\
\hline \multirow[t]{9}{*}{ High Frequency } & $\begin{array}{l}\text { Gender } \\
\text { male } \\
\text { female } \\
\text { not reported }\end{array}$ & $\begin{array}{c}134(56 \%) \\
98(41 \%) \\
6(3 \%)\end{array}$ \\
\hline & hypertension & $38(16 \%)$ \\
\hline & nicotine consumption & $27(11 \%)$ \\
\hline & stress & $76(32 \%)$ \\
\hline & hyperlipedemia & $9(4 \%)$ \\
\hline & $\begin{array}{l}\text { Cardiovascular diseases } \\
\text { (myocardial infarction, stroke) }\end{array}$ & $93(39 \%)$ \\
\hline & diabetes mellitus & $10(4 \%)$ \\
\hline & allergies & $16(7 \%)$ \\
\hline & skin diseases & $6(3 \&)$ \\
\hline \multirow[t]{9}{*}{ Pantonal } & $\begin{array}{l}\text { gender } \\
\text { male } \\
\text { female } \\
\text { not reported }\end{array}$ & $\begin{array}{c}55(52 \%) \\
48(46 \%) \\
2(2 \%) \\
\end{array}$ \\
\hline & hypertension & $19(18 \%)$ \\
\hline & nicotine consumption & $8(8 \%)$ \\
\hline & stress & $33(31 \%)$ \\
\hline & hyperlipedemia & $4(4 \%)$ \\
\hline & $\begin{array}{l}\text { Cardiovascular diseases } \\
\text { (myocardial infarction, stroke) }\end{array}$ & $3(3 \%)$ \\
\hline & diabetes mellitus & $6(6 \%)$ \\
\hline & allergies & $10(10 \%)$ \\
\hline & skin diseases & $2(2 \%)$ \\
\hline
\end{tabular}

Values as number of patients and percent. (a)

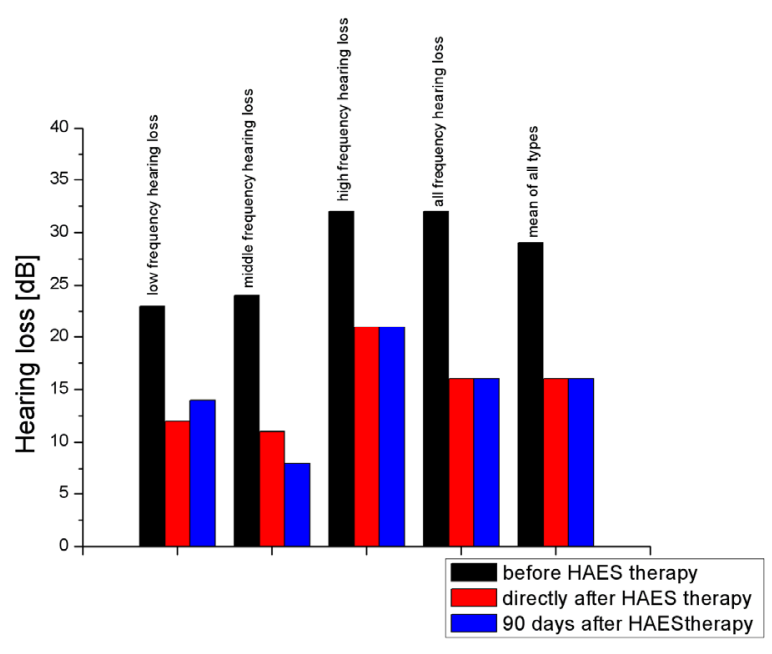

(b)

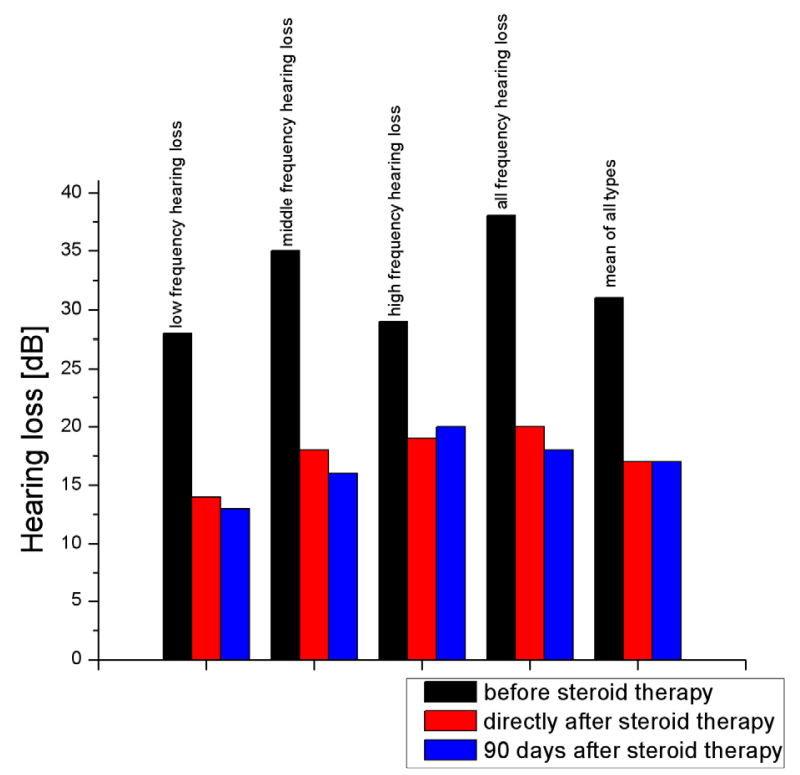

(c)

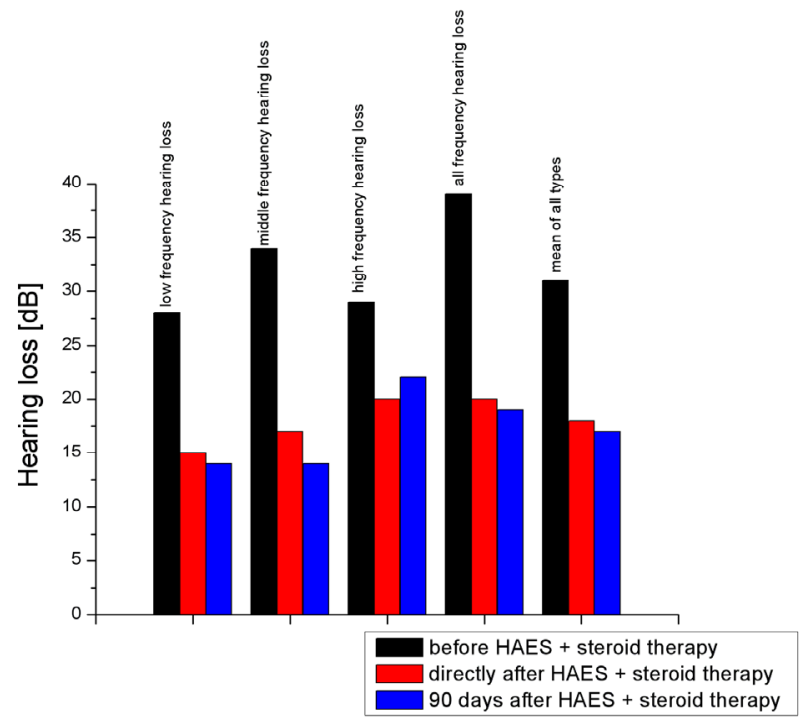

Fig. (3). (a-c) Various hearing loss types before and after therapy with the described therapeutic agents. 
modalities were described and implemented under the hypothesis of vascular pathology of hearing loss in recent years. It was thus far not possible to prove the success of these therapies, since there are only very few clinical data which represent the remission rates of various types of hearing loss and therapeutic modalities while taking the timerelated therapeutic window into account. The insufficient study situation may be due to the fact that the numerous applied therapy forms are very difficult to compare, firstly since different diagnostic criteria are used in most cases and secondly since the rate of spontaneous remissions is very high. This is stated as ranging from $40 \%$ [6] to $89 \%$ [7] for a follow-up observation period of 1 to 2 years. For these reasons, the retrospective assessment was brought into existence in order to allow a better assignment of questions regarding associations between pathogenesis and therapy of hearing loss in the sense of data recorded without time limits using large case numbers of documented demographic, clinical and therapeutic data.

Arterial hypertension is a recognized risk factor for cardiocirculatory disorders such as myocardial infarction or stroke. In the patient collective examined in the present study, this occurred with a frequency of $16 \%$ and therefore lies within the Middle European illness frequency of 10-20 $\%$ of the overall population. The other risk factors such as hyperlipidemia, diabetes mellitus and nicotine consumption are also within the average of Western countries among our patients, so that an above average cardiovascular risk profile could not be determined in patients with hearing loss. This is furthermore confirmed by the fact that the comorbidity of other cardiovascular disorders such as myocardial infarction and apoplexy is also not above average in the patient collective examined by us. Nonetheless, a clinical study in Taiwan [8] showed that patients have a significantly higher risk of stroke after hearing loss as compared to patients without hearing loss. The inclusion of further patients will clarify whether such an observation also applies to Germany.

A therapy form which is commonly used in Europe consists of hypervolemic hemodilution by means of plasma replacement products. The use of plasma expanders increases the circulating blood volume and simultaneously reduces the relative share of the cellular part of the blood. The consequence is an increased heart time volume with simultaneously improved flow attributes of the whole blood. The objective of therapeutic improvement of the blood's flowing behavior is to improve the microcirculation, since this is required for optimal supply of oxygen and energy carriers to the inner ear as well as removal of metabolic end products. Hydroxyethyl starch solutions find particular use in clinical practice. Aside from the effect of hemodilution, HES also brings about a reduction of inflammation signs and endothelial cell activation [9]. Overall, no study which contained at least 50 rheologically treated patients showed a hearing gain or a higher remission rate as compared to the control group which was treated with placebo. Likewise, no therapeutic advantage was shown thus far through a combination of various active substances. However, several studies $[10,11]$ were able to confirm that patients with an elevated risk of not obtaining remission (arterial hypertension, therapy started after more than 2 days) profited from infusion therapy with HES. If one now considers the prospective, randomized studies, that is, those with relatively high evi- dence, HES shows a remission rate of $55-79 \%$ and a hearing gain of $10-14 \mathrm{~dB}$ in the high tone range, as well as $18-$ $25 \mathrm{~dB}$ in the mid-range and/or low tone range. A study which was retrospectively implemented on 603 patients [12] also showed a hearing gain of approximately $18 \mathrm{~dB}$ in the low tone range after sole infusion therapy; mid-range gain was $16 \mathrm{~dB}$ and $24 \mathrm{~dB}$ were gained in the high frequency range. These parameters match the patients studied by us, however they also match the rate of spontaneous remission under placebo therapy. In the present study, HES showed average improvements of approximately $14 \mathrm{~dB}$ in the low and middle frequency ranges and approximately $11 \mathrm{~dB}$ in the high frequency range.

As a side effect of therapy with HES, a dosage dependent, long lasting, but reversible itching sensation has been described. With high dosing of HES, cumulative embedment of hydroxyethyl starch of more than $180 \mathrm{~g}$ into the reticuloendothelial system is discussed as causative. None of the 470 patients who were treated with HES in our study reported pruritus at the 90 day follow-up examination. These data match those of Alexiou [12] who likewise did not report any cases of HES intolerance, while side effects were stated in the glucocorticoid group, so that the side effects were higher overall in the group which was treated with steroids.

The effect of glucocorticoids is based on an antiinflammatory and swelling-reducing effect with the assumption that an inflammation or immune system modulated reaction on the inner ear may be causative for the development of the hearing loss. The effect which reduces swelling as well as the increased response to catecholamines is also intended to increase cochlear blood flow. As for rheological infusion therapy, there are also few placebo controlled clinical studies about the effectiveness of steroid therapy in the treatment of idiopathic hearing loss. Existing studies furthermore show non-uniform results, so that a meta-analysis which was published in 2006 [13] herein also shows the unproven effect of cortisone. In sole therapy with steroids, Mattox and Simmons [14] showed a remission rate of $56 \%$ which was, however, also obtained in the comparison group without therapy. Contrary to this, the double blind, randomized study by Wilson et al. [15] showed a remission rate of $61 \%$ in the steroid group as compared to $32 \%$ in the placebo group and $56 \%$ in the zero therapy group. Investigations of Chen et al. are in line with this study [16]. In the observation implemented by us, sole therapy with steroids showed average improvements of approximately $15 \mathrm{~dB}$ in the low and mid-range tone ranges and approximately $10 \mathrm{~dB}$ in the high tone range. These parameters are within the range of sole therapy with rheologics. Even in the combination of rheological therapy with steroids, the patient collective which was examined by us showed no therapeutic advantage as compared to sole steroid therapy. This observation is contrary to the results of a retrospective cohort study [12] on 603 patients which showed a significantly better recovery of hearing in 302 patients through combination of rheologics with steroids in pancochlear or low tone hearing loss as compared to sole infusion therapy. The obtained average hearing gain in this study of $24 \mathrm{~dB}$ in the low tone, $20 \mathrm{~dB}$ in the mid-tone and $12 \mathrm{~dB}$ in the high tone range was significantly better in combination than $18 \mathrm{~dB}, 16 \mathrm{~dB}$ and $14 \mathrm{~dB}$ through sole therapy with rheologics. In the patient collective studied by us, the hearing gain of $15 \mathrm{~dB}$ in the low tone, 
$17 \mathrm{~dB}$ in the mid-tone and $9 \mathrm{~dB}$ in the high tone range was equal for both individually applied therapeutic modalities.

The time-wise therapeutic window of hearing loss is subject to highly controversial discussion and differs between 14 and 42 days. In order to be able to make more precise statements in this regard for therapy, we considered remission rates according to the age of the hearing loss until day 90 . Herein it is shown that the hearing loss drastically lessens after therapy in a period longer than two weeks. However it is also necessary to consider that the absolute hearing loss after two weeks is also considerably less due to the spontaneous remission rate. Therapy of hearing loss was able to gain an average improvement of $42 \%$ within the first two weeks. This parameter falls to an average of $21 \%$ when observing the time periods between 14 and 90 days. This observation matches the results of other clinical studies which show that the time until the commencement of therapy must be regarded as a prognostic factor for the remission rate [3. 11. 17-19].

\section{SUMMARY}

Standardized documentation of the treatment of hearing loss patients is able to provide important knowledge for the treatment of hearing loss patients. This retrospective assessment, which is not limited in terms of time, will use its large case numbers in the future to identify subgroups of hearing loss which can be used to define clearly delimitable criteria for differing therapeutic modalities in the sense of differential therapy.

\section{REFERENCES}

[1] Olzowy B, Osterkorn D, Suckfull M. The incidence of sudden hearing loss is greater than previously assumed. MMW Fortschr Med 2005; 147: 37-8.

[2] Miller JM, Ren TY, Nuttall AL. Studies of inner ear blood flow in animals and human beings. Otolaryngol Head Neck Surg 1995; 112: 101-13.

[3] Byl FM, Jr. Sudden hearing loss: eight years' experience and suggested prognostic table. Laryngoscope 1984; 94: 647-61.
[4] Michel O, Jahns T, Joost-Enneking M, Neugebauer P, Streppel M, Stennert E. The Stennert antiphlogistic-rheologic infusion schema in treatment of cochleovestibular disorders. HNO 2000; 48: 182-8.

[5] Suckfull M. Fibrinogen and LDL apheresis in treatment of sudden hearing loss: a randomised multicentre trial. Lancet 2002; 360: 1811-7.

[6] Laird N, Wilson WR. Predicting recovery from idiopathic sudden hearing loss. Am J Otolaryngol 1983; 4: 161-4.

[7] Heiden C, Porzsolt F, Biesinger E, Hoing R. Spontaneous remission of sudden deafness. HNO 2000; 48: 621-3.

[8] Lin HC, Chao PZ, Lee HC. Sudden sensorineural hearing loss increases the risk of stroke: a 5-year follow-up study. Stroke 2008; 39: 2744-8.

[9] Boldt J, Ducke M, Kumle B, Papsdorf M, Zurmeyer EL. Influence of different volume replacement strategies on inflammation and endothelial activation in the elderly undergoing major abdominal surgery. Intensive Care Med 2004; 30: 416-22.

[10] Desloovere C, Meyer-Breiting E, von IC. Randomized doubleblind study of therapy of sudden deafness: initial results. HNO 1988; 36: 417-22.

[11] Klemm E, Bepperling F, Burschka MA, Mosges R. Hemodilution therapy with hydroxyethyl starch solution $(130 / 0.4)$ in unilateral idiopathic sudden sensorineural hearing loss: a dose-finding, double-blind, placebo-controlled, international multicenter trial with 210 patients. Otol Neurotol 2007; 28: 157-70.

[12] Alexiou C, Arnold W, Fauser C, et al. Sudden sensorineural hearing loss: does application of glucocorticoids make sense? Arch Otolaryngol Head Neck Surg 2001; 127: 253-8.

[13] Wei BP, Mubiru S, O'Leary S. Steroids for idiopathic sudden sensorineural hearing loss. Cochrane Database Syst Rev 2006;(1):CD003998.

[14] Mattox DE, Simmons FB. Natural history of sudden sensorineural hearing loss. Ann Otol Rhinol Laryngol 1977; 86: 463-80.

[15] Wilson WR, Byl FM, Laird N. The efficacy of steroids in the treatment of idiopathic sudden hearing loss. A double-blind clinical study. Arch Otolaryngol 1980; 106: 772-6.

[16] Chen CY, Halpin C, Rauch SD. Oral steroid treatment of sudden sensorineural hearing loss: a ten year retrospective analysis. Otol Neurotol 2003; 24: 728-33.

[17] Byl FM. Seventy-six cases of presumed sudden hearing loss occurring in 1973: prognosis and incidence. Laryngoscope 1977; 87: 817-25.

[18] Zadeh MH, Storper IS, Spitzer JB. Diagnosis and treatment of sudden-onset sensorineural hearing loss: a study of 51 patients. Otolaryngol Head Neck Surg 2003; 128: 92-8.

[19] Xenellis J, Karapatsas I, Papadimitriou N, et al. Idiopathic sudden sensorineural hearing loss: prognostic factors. J Laryngol Otol 2006; 120: 718-24. 